\title{
Relationships between statistics of rainfall extremes and mean annual precipitation: an application for design-storm estimation in northern central Italy
}

\author{
G. Di Baldassarre, A. Castellarin, and A. Brath \\ DISTART, Faculty of Civil Engineering, University of Bologna, Bologna, Italy \\ Received: 16 September 2005 - Published in Hydrol. Earth Syst. Sci. Discuss.: 16 November 2005 \\ Revised: 19 April 2006 - Accepted: 26 July 2006 - Published: 21 August 2006
}

\begin{abstract}
Several hydrological analyses need to be founded on a reliable estimate of the design storm, which is the expected rainfall depth corresponding to a given duration and probability of occurrence, usually expressed in terms of return period. The annual series of precipitation maxima for storm duration ranging from $15 \mathrm{~min}$ to 1 day, observed at a dense network of raingauges sited in northern central Italy, are analyzed using an approach based on L-moments. The analysis investigates the statistical properties of rainfall extremes and detects significant relationships between these properties and the mean annual precipitation (MAP). On the basis of these relationships, we developed a regional model for estimating the rainfall depth for a given storm duration and recurrence interval in any location of the study region. The applicability of the regional model was assessed through Monte Carlo simulations. The uncertainty of the model for ungauged sites was quantified through an extensive crossvalidation.
\end{abstract}

\section{Introduction}

Design storm are usually estimated by regional frequency analysis of rainfall extremes when there are no measured data for the location of interest, or when data record lengths are short compared to the recurrence interval of interest (Brath et al., 1998; Faulkner, 1999; Brath and Castellarin, 2001).

This study analyses the annual series of precipitation maxima observed at a dense raingauge network located in a wide geographical area of northern central Italy. Several regional frequency analyses of rainfall extremes were performed over the study area analysed here in (Franchini and Galeati, 1994; Brath et al, 1998). These studies proposed subdivisions of the region into homogeneous climatic regions, within which

Correspondence to: G. Di Baldassarre

(giuliano.dibaldassarre@ mail.ing.unibo.it) the statistics of rainfall extremes for a given duration are assumed to be constant (Brath and Castellarin, 2001). This assumption contrasts with the findings of other studies, which show that the statistics of rainfall extremes vary systematically with location (Schaefer, 1990; Alila, 1999; Brath et al., 2003). These studies also identified statistically significant relationships between these statistics and the mean annual precipitation (MAP), which was used as a surrogate of geographical location.

For instance, Schaefer (1990) analysed the rainfall series collected at hundreds of gauges located in Washington State (USA) and showed that the coefficients of variation and skewness of rainfall extremes tend to decrease as the local value of MAP increases. Alila (1999) studied the Canadian raingauge network and detected analogous relationship between the L-coefficients of variation, L- $C v$ (for a definition of the coefficients see e.g. Hosking and Wallis, 1997). Brath et al. (2003) identified a similar behaviour of L-Cv and L-Cs (L-coefficients of skewness) of rainfall extremes for the same study area considered herein and storm-duration between 1 and $24 \mathrm{~h}$. We investigated further the applicability of these outcomes to the study region for sub-hourly storm-duration and, on the basis of the findings obtained, we formalised the relationship between L-statistics of rainfall extremes and MAP through a Horton-type curve (Horton, 1939). Once assessed the applicability of the proposed mathematical expression through an original and objective Monte Carlo simulation experiment, we developed a regional model for estimating design storms for storm duration from $15 \mathrm{~min}$ to 1 day in any location of the study area and we quantified the uncertainty of the regional model for ungauged sites through an extensive cross-validation.

Published by Copernicus GmbH on behalf of the European Geosciences Union. 


\section{Index storm procedure}

The design of numerous hydraulic engineering structures and several hydrological applications need to be based on an estimate of a design storm, which is the expected rainfall depth $h(d, T)$ corresponding to a given duration $d$ and probability of occurrence, usually expressed in terms of return period $T$.

A regional frequency analysis can be implemented using the index storm procedure (Dalrymple, 1960; Brath et al., 2003). The index storm methodology is based on the identification of homogeneous groups of sites for which $h(d, T)$ can be expressed as the product of two terms, as follows:

$h(d, T)=m_{d} h^{\prime}(d, T)$

these two terms are a scale factor $m_{d}$, which is called index storm, and a dimensionless growth factor $h^{\prime}(d, T)$, which describes the relationship between the dimensionless storm and the recurrence interval. The index storm, usually assumed equal to the mean of annual rainfall maxima of duration $d$, is site dependent; while the growth factor is assumed to be valid for the entire homogeneous group of basins.

\subsection{Growth factor estimation}

The classical implementation of the index flood procedure (or index storm if reference is made to rainfall extremes) is based on the most restrictive fundamental hypothesis of existence of homogeneous regions within which the statistical properties of dimensionless rainfall extremes (see e.g., Franchini and Galeati, 1994; Brath et al., 1998) do not vary with location (i.e., coefficients of variation and skewness, or equivalently $\mathrm{L}-C v$ and $\mathrm{L}-C s$, are constant).

Nevertheless, since the original procedure was introduced (see e.g. Dalrymple, 1960) several extensions and evolutions were proposed, which partly relax this fundamental hypothesis. An example is the hierarchical application of the index flood hypothesis, where the statistics of increasing order are constant within a set of nested regions, the larger the order of the statistics, the larger the region (see e.g. Gabriele and Arnell, 1991). Another relevant example of evolution of the original hypothesis is the Region of Influence approach (e.g., Burn, 1990; Castellarin et al., 2001), which adopts the concept of homogeneous pooling groups of sites as opposed to homogeneous geographical regions. We present a regional model that can be considered to be an extension of the index flood model as well. Similarly to what originally proposed in Schaefer (1990) and Alila (1999), we assume that a homogeneous region, within which $\mathrm{L}-C v$ and $\mathrm{L}-C s$ are constant, is a group of climatically homogeneous sites, within which the variability of MAP is very limited.

The study described in this paper investigates the applicability of the findings of Schaefer (1990) and Alila (1999) in a large geographical area of northern central Italy. The analysis considers annual series of precipitation maxima for storm duration from 15 min to 1 day that were observed by a dense raingauge network.

We characterised the regional frequency regime of rainfall extremes over the study area using the L-moments as suggested by Hosking and Wallis (1997). The L-moments are analogous to the conventional moments, but they have the theoretical advantages of being able to characterize a wider range of distributions and, when estimated from a sample, of being more robust to the presence of outliers in the data. Hosking and Wallis (e.g., 1997) also point out that Lmoments are less subject to bias in estimation than conventional moments. Nevertheless, Klemes (2000) considered the lack of sensitivity to outliers to be a disadvantage in the use of L-moments. The recent study (Klemes, 2000) argues that high outliers in a hydrologic data series are important for extrapolating to large return period events as they control the right tail of the frequency distribution.

The growth factor estimation was performed by using the Generalised Extreme Value (GEV) distribution (Jenkinson, 1955). The GEV distribution subsumes all three different extreme-value distributions (i.e., EV type I, II and III), to which the largest/smallest value from a set of independent and identically distributed random variables asymptotically tends. Consistently, several recent regional analyses showed that the GEV distribution is a suitable statistical model for representing the frequency regime of rainfall extremes over the whole study area (see e.g., Franchini and Galeati 1994; Brath et al. 1998).

The CDF (cumulative distribution function) of the GEV distribution is written as:

$$
F_{X}(x)=\exp \left\{-\left[1-\frac{k(x-\xi)}{\alpha}\right]^{1 / k}\right\}, \quad \text { for } \quad k \neq 0
$$

and

$F_{X}(x)=\exp \left\{-\exp \left[-\frac{(x-\xi)}{\alpha}\right]\right\}, \quad$ for $\quad k=0$

while the quantile $x(F)$ can be written as:

$x(F)=\xi+\alpha\left\{1-(-\log F)^{k}\right\} / k$, for $\quad k \neq 0$

and

$x(F)=\xi+\alpha \log (-\log F)$, for $k=0$

where $\xi, \alpha$, and $k$ are the distribution parameters. As shown by Eqs. (2b) and (3b), when $k=0$ the GEV distribution is equal to the Gumbel distribution. Combining formulations (2) and (3) can be obtained the relations for the regional growth factor, replacing the variable $X$ with the dimensionless variable $X^{\prime}=X / \mu$ and the parameters $\alpha, \xi$ and $k$ with the regional parameters $\alpha^{\prime}=\alpha / \mu, \xi^{\prime}=\xi / \mu$ and $k^{\prime}=k$, where the expected value $\mu$ is written as:

$\mu=\xi+\left(\frac{\alpha}{k}\right)[1-(1+k)]$. 


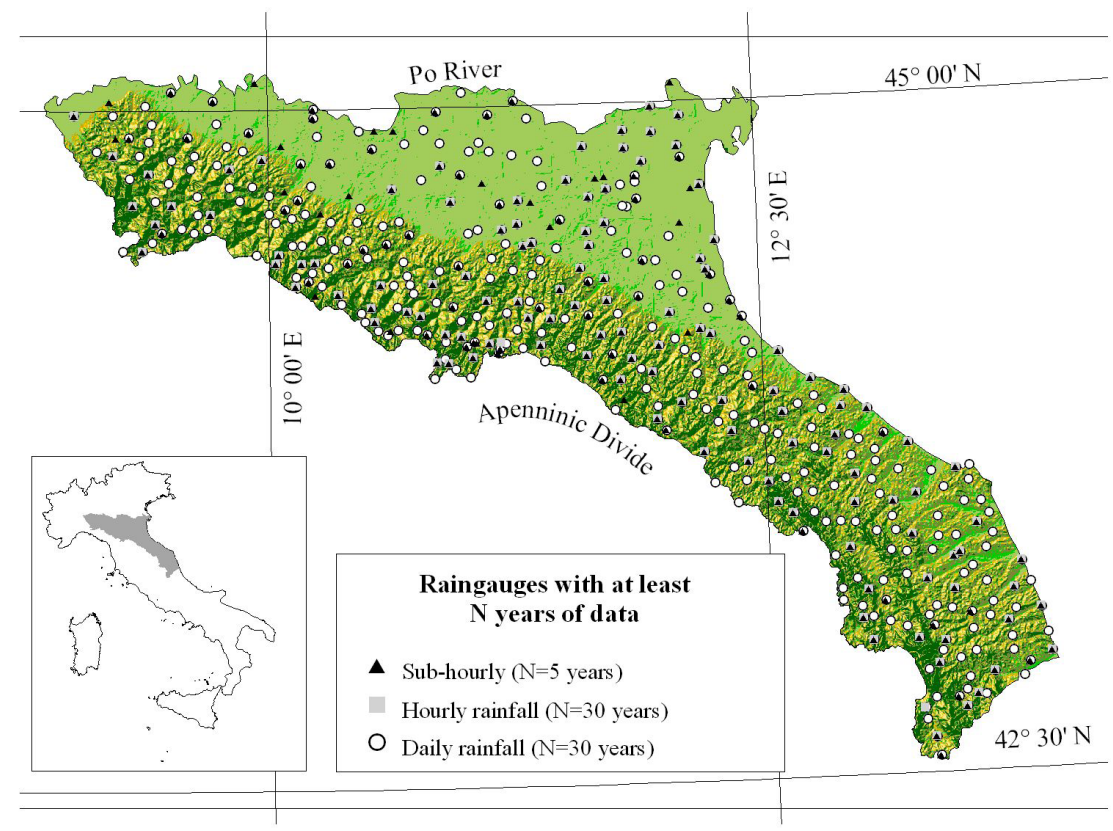

Fig. 1. Study area and location of raingauges for different values of the minimum record length.

The parameters $\alpha^{\prime}, \xi^{\prime}$ and $k^{\prime}$ can be estimated through the regional procedure based on L-moments, using the following relations (Hosking and Wallis, 1997):

$k^{\prime} \approx 7.8590 c+2.9554 c^{2}$, with $c=\frac{2}{3+\mathrm{L}-C s_{R}}-\frac{\log 2}{\log 3}$

and

$$
\alpha^{\prime}=\frac{\mathrm{L}-C v_{R} k^{\prime}}{\left(1-2^{-k^{\prime}}\right) \Gamma\left(1+k^{\prime}\right)}, \xi^{\prime}=1-\alpha^{\prime}\left\{1-\Gamma\left(1+k^{\prime}\right)\right\} / k^{\prime}
$$

where $\mathrm{L}-C v_{R}$ and $\mathrm{L}-C s_{R}$ are, respectively, the 2nd and 3rd order standardised regional L-moments. Hosking and Wallis (1997) illustrate in detail the general procedure for computing $\mathrm{L}-C v_{R}$ and $\mathrm{L}-C s_{R}$ for a particular pooling-group of sites (i.e., a geographical region or a set of raingauges). Our study develops a regional model for estimating $\mathrm{L}-C v_{R}$ and $\mathrm{L}-C s_{R}$ for the storm duration of interest from the local MAP value (see Sect. 4).

\subsection{Index storm estimation}

The estimation of the index storm $m_{d}$ can be usually obtained by computing the sample mean of annual rainfall maxima of duration $d$. If there are no measured data for the site of interest the index storm estimation can be obtained from the observations collected at neighbouring sites through a suitable spatial interpolator (Brath and Castellarin, 2001; Castellarin and Brath, 2002). The uncertainty associated with this estimation procedure is discussed in detail in Sect. 5.2.

\section{Study area and local regime of rainfall extremes}

The study area includes the administrative regions of EmiliaRomagna and Marche, in northern central Italy, and occupies $35800 \mathrm{~km}^{2}$. The area is bounded by the Po River on the north, the Adriatic Sea on the east, and the Apennine divide on the southwest (see Fig. 1). The north-eastern portion of the study region is mainly flat, while the south-western and coastal parts are predominantly hilly and mountainous.

The database of extreme rainfall consists of the annual series of precipitation maxima with duration $d$ equal to 15 and $30 \mathrm{~min} ; 1,3,6,12$ and $24 \mathrm{~h}$ and 1 day (i.e., from 09:00 a.m. to 09:00 a.m. of the following day) that were obtained for a dense network of rain gauges from the National Hydrographical Service of Italy (SIMN) in the period 1935-1989. The available rainfall data are summarised in Table 1.

As Table 1 shows, we considered all available series of sub-hourly rainfall with at least 5 years of measure $(\mathrm{N} \geq 5)$. This criterion reflects our intention of incorporating into the analysis as much information as possible. The raingauge network for sub-hourly storm duration is more recent and sparser than the network for hourly and daily storm duration (see Table 1). In order to reduce the negative effects of sampling variability for short series we characterised the statistics of rainfall extremes locally at each site through sample Lmoments estimators, as they tend to be less biased than sample estimators of traditional statistical moments for orders 2 and higher and small samples (e.g., Hosking, 1990; Hosking and Wallis, 1997). For the same reason, as suggested for instance in Hosking and Wallis (1997), we characterised the frequency regime of rainfall extremes at a regional scale 
Table 1. Study Area: Number of Raingauges and Annual Maximum Rainfall Data.

\begin{tabular}{llll}
\hline Duration & Criterion & Number of Gauges & Station-year of data \\
\hline daily & $\mathrm{N} \geq 30$ & 394 & 20557 \\
hourly & $\mathrm{N} \geq 30$ & 125 & 5945 \\
30 min & $\mathrm{N} \geq 5$ & 186 & 3430 \\
15 min & $\mathrm{N} \geq 5$ & 152 & 1810 \\
\hline
\end{tabular}

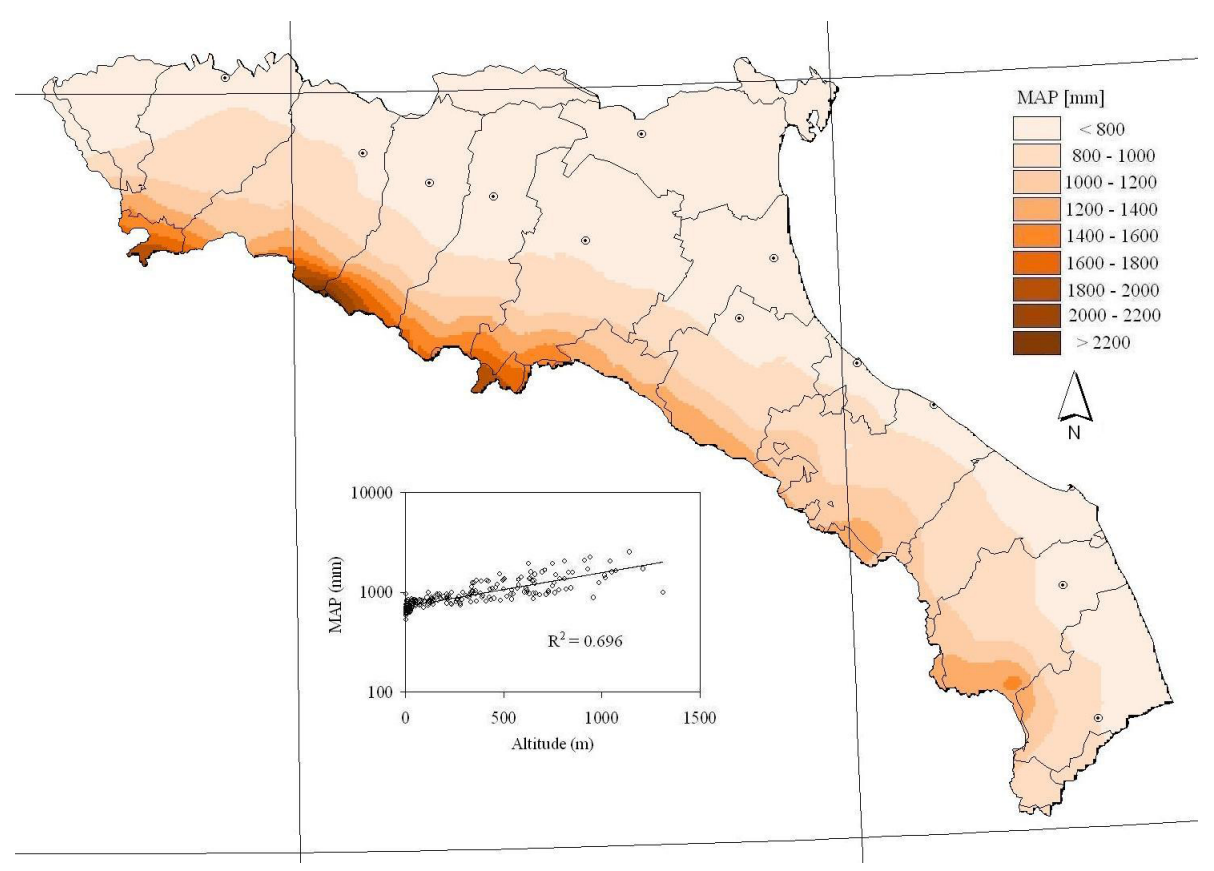

Fig. 2. Mean annual precipitation MAP (mm). MAP versus Altitude (m a.s.1.).

(i.e., for a group of raingauges) by weighting each sample L-moment proportionally to the sample length.

A regional analysis of the dates of occurrence of shortduration rainfall extremes (i.e., 1 or $3 \mathrm{~h}$ ) pointed out significant consistency and a mean timing which varied between the end of July and the beginning of August for the entire study area (Castellarin and Brath, 2002). This is consistent with the observation that in the study area the hourly rainfall extremes are almost invariantly summer showers generated by local convective cells. The dates of occurrence of longduration rainfall extremes (i.e., $24 \mathrm{~h}$ or 1 day) showed less regularity and a mean timing that ranges between the beginning of September and the beginning of November.

MAP varies on the study region from about 500 to $2500 \mathrm{~mm}$. Altitude is the factor that most affects the MAP (see Figs. 1 and 2), which exceeds $1500 \mathrm{~mm}$ starting from altitudes higher than $400 \mathrm{~m}$ a.s.l. and exhibits the highest values along the divide of the Apennines.

The diagram of L-moment ratios (see e.g., Hosking and Wallis, 1993) reported in Fig. 3 shows that the theoretical re- lationship between L-skewness (L-Cs) and L-kurtosis (L-Ck) for the GEV distribution is very close to the regional L-Cs and $\mathrm{L}-\mathrm{Ck}$ values for all storm duration of interest, therefore indicating that the GEV distribution is a suitable parent distribution.

The study investigates the applicability of the finding of Schaefer (1990) and Alila (1999) in this particular context, making use of a raingauge network with a higher resolution than the networks considered in the above mentioned papers. The variability of the sample L-moment ratio (Hosking, 1990) of skewness and variation was examined against the variability of MAP. Figure 4 shows that the values of L$C v$ and $\mathrm{L}-C s$ of rainfall extremes tend to increase when the MAP value decreases, confirming some of the results pointed out by Alila (1999). 


\section{Regional model}

\subsection{Climatically homogeneous pooling-groups}

As previously mentioned, the estimation of the dimensionless growth factor $h^{\prime}(d, T)$ can also be carried out by dispensing with the traditional subdivision of the study area into homogeneous regions. In fact, the papers of Schaefer (1990) and Alila (1999) point out that the statistics of rainfall extremes vary systematically with location, showing that all hypotheses of subdivisions into geographical regions lack physical basis. These studies identified statistically significant relationships between these statistics and the MAP, which was used as a surrogate of geographical location.

We developed frequency analysis of rainfall extremes using the MAP values and the L-moments $\mathrm{L}-\mathrm{Cs}$ and $\mathrm{L}-\mathrm{C} v$ (Hosking and Wallis, 1997), for any considered duration ( $d$ equal to 15 and $30 \mathrm{~min} ; 1,3,6,12$ and $24 \mathrm{~h} ; 1$ day). The analysis points out that the L-Cv values tend to decrease as the local value of MAP increases (see Fig. 4); for storm duration from $15 \mathrm{~min}$ to $6 \mathrm{~h}$ the L-Cs values are approximately constant with the geographic position (identified with the MAP value); for longer storm duration the L-Cs values tend to decrease as the local value of MAP increases.

We designed and performed a statistical homogeneity test which uses the heterogeneity measures proposed by Hosking and Wallis (1993) but also incorporates the findings of Schaefer (1990) and Alila (1999) by grouping the stations that show similar MAP values. The statistical test (see also Appendix) assesses the homogeneity of a group of stations according to 2 measures of dispersion of the sample Lmoments:

1. $\mathrm{H}(1)$, that focuses on the dispersion of sample $\mathrm{L}-\mathrm{Cv}$ values;

2. $H(2)$, that focuses on the combined dispersion of sample $\mathrm{L}-C v$ and $\mathrm{L}-C s$ values.

Hosking and Wallis (1993) suggested that the region or group of sites should be considered as acceptably homogeneous if $\mathrm{H}<1$; possibly heterogeneous if $1 \leq \mathrm{H}<2$, and definitely heterogeneous if $\mathrm{H} \geq 2$.

The homogeneity testing has been developed in the following steps: the set of $\mathrm{N}$ raingauges was sorted in ascending order of MAP values; with this ordered set, the $N-n+1$ subsets were identified considering, each time, the $n$ closer station in terms of MAP (with $n=15,30,60$ ); the $\mathrm{H}(1)$ values were calculated for each group of 15 and 30 stations, while the $\mathrm{H}(2)$ values were calculated for the groups with 30 and 60 stations. The $H(1)$ and $H(2)$ values were assigned to the average MAP value of the subset and the behaviour of $\mathrm{H}(1)$ and $\mathrm{H}(2)$ values as a function of MAP was then analysed. The different numbers of raingauges considered for the homogeneity testing (i.e., 15 and 30 for $\mathrm{H}(1)$ and 30 and 60 for $\mathrm{H}(2)$ ) reflect two different aspects. First, higher order L-moments tend to be
- Gumbel

- Generalized Extreme Value

-.. Generalized Pareto

-.... Generalized Logistic

O Regional sample L moments

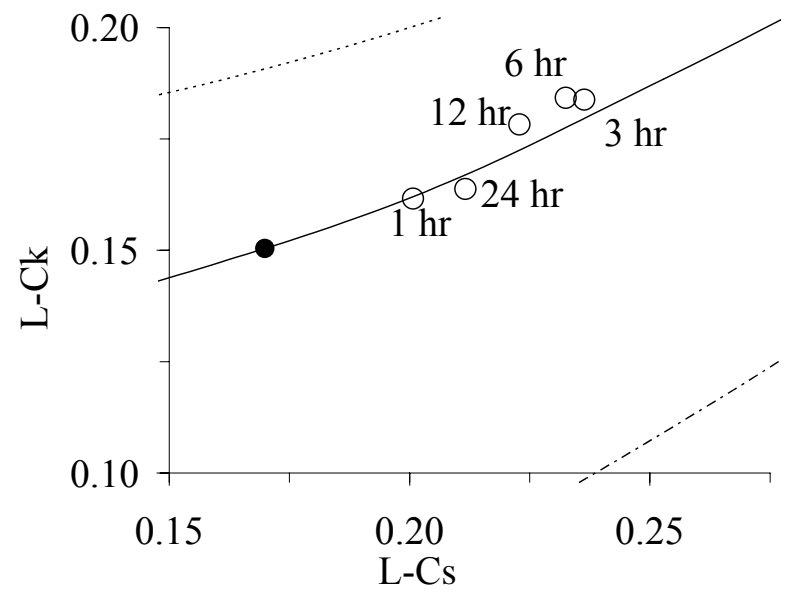

Fig. 3. Diagram of L-moment ratios for the application data.

more homogeneous in space than the lower order ones (see e.g., Hosking and Wallis, 1997), therefore pooling-groups of sites for which the homogeneity is assessed in terms of L-Cv and L-Cs (i.e., use of $\mathrm{H}(2)$ ) may be lager than pooling-groups for which the homogeneity is assessed in terms of L-Cv only (i.e., use of $\mathrm{H}(1)$ ). Second, heterogeneity measures such as $\mathrm{H}(1)$ and $\mathrm{H}(2)$ are better at indicating heterogeneity in large regions, while have a tendency to give false indications of homogeneity for small regions, therefore pooling groups should be as larger as possible. This analysis (see Fig. 5) shows that the subsets identified according to the MAP value are generally acceptably homogeneous, whereas the $\mathrm{H}(1)$ value for the whole study region is equal to 3.41 while the $\mathrm{H}(2)$ value is equal to 1.73. Also, Fig. 5 shows that the $\mathrm{H}(1)$ and $\mathrm{H}(2)$ values, quantifying the homogeneity degree, are significantly MAP independent. This result underlines the advantage of using MAP a surrogate of geographical location.

4.2 Empirical regional model for estimating the L-Cv and $\mathrm{L}-C s$

After testing the regional homogeneity of the climatically similar group of sites (identified according to MAP values) the analysis has been directed to develop an empirical regional model for estimating the regional statistics L-Cs and L-C $v_{R}$, which can then be used for estimating the GEV parameters.

In detail, we formalised the relationships between Lmoments and MAP illustrated in Fig. 4 using a Horton-type curve::

$\mathrm{L}-C x(\mathrm{MAP})=a+(b-a) \times \exp (-c \times \mathrm{MAP})$, 

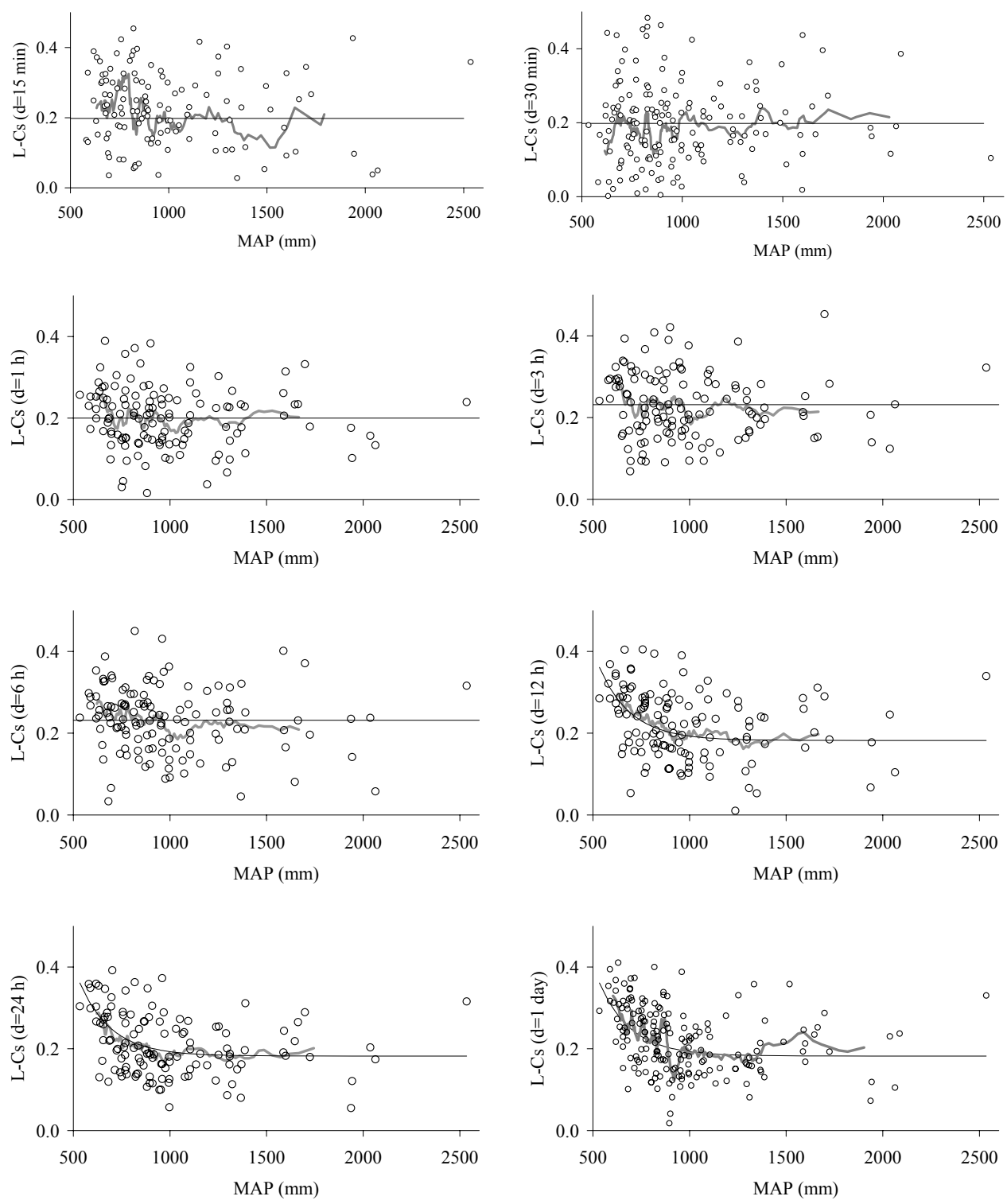

Fig.4a. Sample L-Cs versus MAP (mm) for durations between $15 \mathrm{~min}$ and 1 day (circles), moving weighted average curves (grey lines) and empirical regional model (black lines).

Table 2. Coefficients $a, b$ and $c$ of regional model (6) for estimating $\mathrm{L}-\mathrm{Cs}_{R}$ and L-Cv $v_{R}$.

\begin{tabular}{lllllll}
\hline \multirow{2}{*}{ Duration } & \multicolumn{3}{c}{ L-CsR } & & \multicolumn{3}{c}{$\mathrm{L}-C v_{R}$} \\
& $a$ & $b$ & $c$ & $a$ & $b$ & $c$ \\
\hline $15 \mathrm{~min}$ & 0.1999 & 0.1999 & 0 & 0.1539 & 0.1539 & 0 \\
$30 \mathrm{~min}$ & 0.1999 & 0.1999 & 0 & 0.1893 & 0.1893 & 0 \\
$1 \mathrm{~h}$ & 0.1999 & 0.1999 & 0 & 0.1978 & 0.6255 & 0.0038 \\
$3 \mathrm{~h}$ & 0.2318 & 0.2318 & 0 & 0.1856 & 0.8352 & 0.0042 \\
$6 \mathrm{~h}$ & 0.2318 & 0.2318 & 0 & 0.1741 & 0.8436 & 0.0042 \\
$12 \mathrm{~h} \leq d \leq 24 \mathrm{~h}, d=1$ day & 0.1824 & 4.7240 & 0.0061 & 0.1706 & 0.7694 & 0.0040 \\
\hline
\end{tabular}



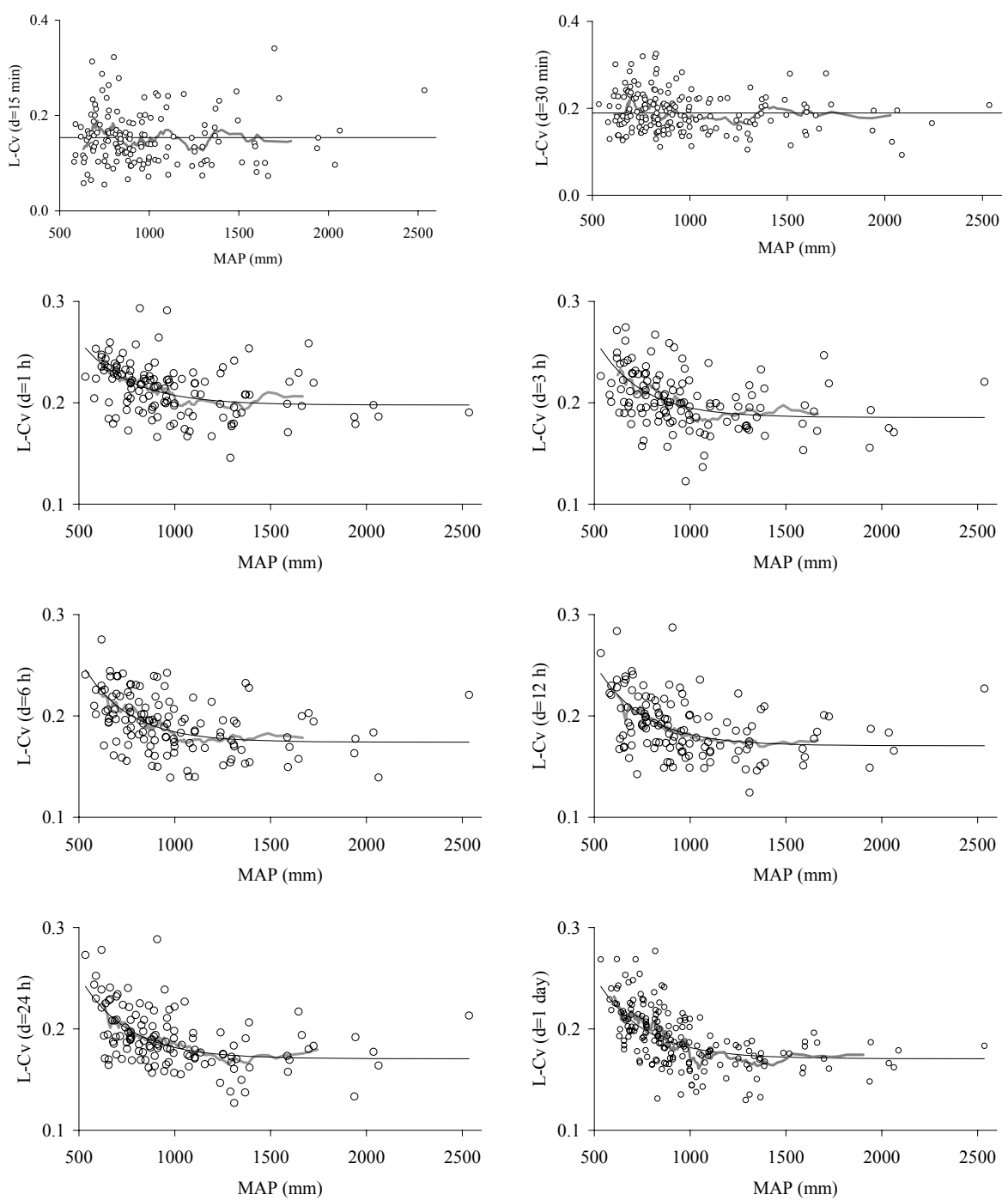

Fig. 4b. Sample L- $C v$ versus MAP (mm) for durations between $15 \mathrm{~min}$ and 1 day (circles), moving weighted average curves (grey lines) and empirical regional model (black lines).

where $\mathrm{L}-C x$ represents a particular regional L-moment (L$C s_{R}$ or L- $C v_{R}$ ), related to the annual maximum series (AMS) of rainfall depth with storm duration $d$, while $a, b, c$, with $0 \leq a \leq b$ and $c \geq 0$, are the parameters of the empirical model that have to be estimated through an optimisation procedure. If $a=b$ and $c=0$, Eq. (6) has a constant value. This is the case in which the particular L-moment is MAP independent.

We performed the identification of parameters $a, b$ and $c$ on the basis of the empirical outcomes illustrated in Fig. 4, also taking into account the conclusions of previous studies performed over the same study region (e.g., Franchini and Galeati, 1994; Brath and Franchini, 1999; Castellarin and Brath, 2002), which can be sketched as follows:

1. L-Cs can be considered to be independent of the geographic location (or MAP) for $\mathrm{d}<6 \mathrm{~h}$;
2. L-Cs can be considered to be independent of geographic location and duration $d$, for $d=15$ and $30 \mathrm{~min}$ and $1 \mathrm{~h}$;

3. $\mathrm{L}-C s$ can be considered to be the same for duration $\mathrm{d}=3$ and $6 \mathrm{~h}$ (Castellarin and Brath, 2002);

4. L-Cv can be considered to be independent of the geographic location (or MAP) for $\mathrm{d}<1 \mathrm{~h}$, with different values for $\mathrm{d}=15$ and $30 \mathrm{~min}$;

5. The relationships between $\mathrm{L}-C s$, or $\mathrm{L}-C v$, and the geographic location (or MAP) identified for daily observations can be used also for $\mathrm{d} \geq 12 \mathrm{~h}$ (Franchini and Galeati,1996; Brath and Franchini 1999; Castellarin and Brath 2002). 

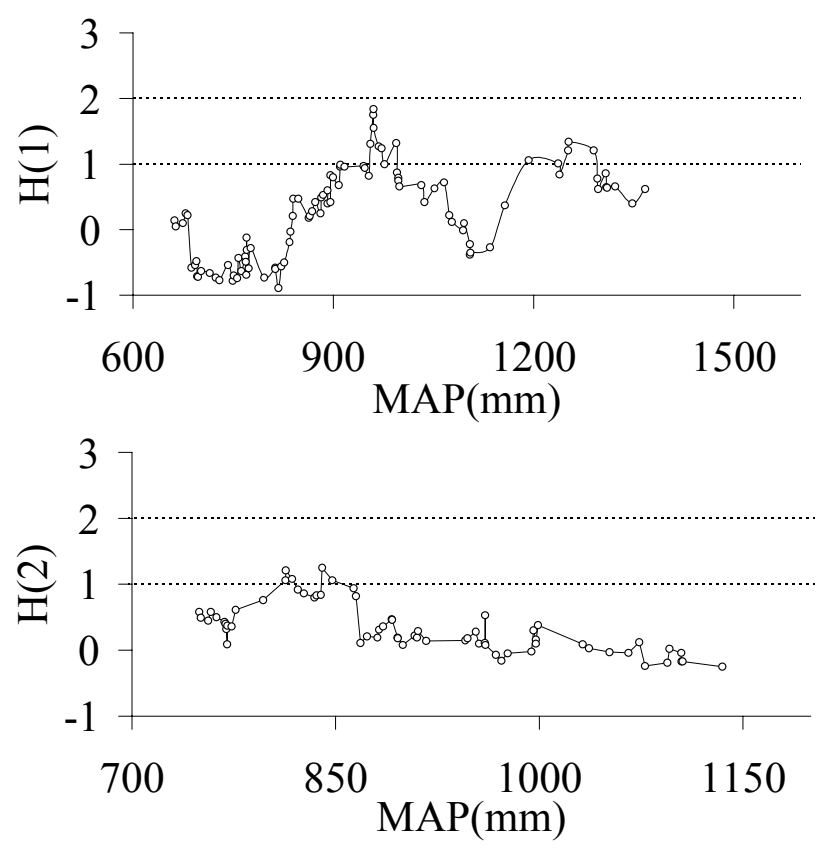

Fig. 5. Heterogeneity measures versus MAP: H(1) values for groups of 30 stations and $d=6 \mathrm{~h}(\mathrm{H}(1)=3.41$ for the entire study region); $\mathrm{H}(2)$ values for groups of 60 stations and $d=24 \mathrm{~h}(\mathrm{H}(2)=1.73$ for the entire study region).

Figure 4 shows the identified empirical regional models, while Table 2, summarises the values of parameters $a, b$ and $c$ for any storm duration. Table 2 shows that, for AMS with storm duration lower than $1 \mathrm{~h}, \mathrm{~L}-C v_{R}$ increases with duration. This outcome confirms the results obtained in previous studies for different geographic area (Alila, 1999).

The overall number of parameters of the regional model might be reduced by looking for scaling relationships in the parameters of the model. With respect to depth-durationfrequency curves, Burlando and Rosso (1996) proposed an approach for limiting the parameterisation requirements by assuming that rainfall depth, once rescaled through a suitable power-law multiplier, follows the same probability distribution for any storm duration. Nevertheless, many authors also showed that this is often violated in practice; rainfall data typically show a transition in their scaling properties for storm duration around $1 \mathrm{~h}$ and shorter (see e.g. Olsson and Burlando, 2002; Marani, 2003). On the basis of the above evidence, the scale invariance assumption was not applied in our study.

The applicability of the identified empirical model was assessed through Monte Carlo simulations. In detail, (a) for any station and for each duration, the regional L-CV and the $\mathrm{L}-C s$ values were calculated as a function of the local MAP value through model (6) with parameters $a, b, c$ listed in Table 2; (b) with these regional L-statistics, regional estimates of the GEV distribution were calculated from the site of inter-
Table 3. Percentage of the sample values of L-Cv and L-Cs lying out side the confidence intervals.

\begin{tabular}{lll}
\hline Significance level & L-Cs & L-Cv \\
\hline $5 \%$ & $4.20 \%$ & $4.80 \%$ \\
$10 \%$ & $8.50 \%$ & $9.80 \%$ \\
\hline
\end{tabular}

est and the considered duration; (c) this probabilistic model was then used to generate synthetic series with length equal to the corresponding historical series; (d) for these synthetic series the sample L-Cv and L-Cs were then calculated. We repeated these steps 5000 times, obtaining 5000 sample Lmoment values, which we finally used to derive the confidence intervals for testing the significance of the empirical model (see Fig. 6).The Monte Carlo simulations test the null hypothesis that the model (6) is able to reproduce the statistical behaviour of rainfall extremes at the 5\% and $10 \%$ significance levels. Table 3 summarises the results obtained with the Monte Carlo analysis, reporting the percentage of sample L-Cv and L-Cs values lying out of confidence intervals. The results indicated that the null hypothesis could not be rejected at 5 and $10 \%$ significance levels as the percentage of $\mathrm{L}-C v$ and $\mathrm{L}-C s$ values lying out side the confidence intervals is less than 5 and $10 \%$, respectively.

\section{Design storm estimation in ungauged sites}

\subsection{Application of the regional model}

In this section we are providing a brief summary illustrating the application of the proposed model to ungauged sites. The model can be applied at any site in the study region for $d$ equal to 15 and $30 \mathrm{~min} ; 1,3,6,12$ and $24 \mathrm{~h}$ and 1 day. For each storm duration $d$ and return period $T$, the design storm $h(d, T)$ can be evaluated as follows:

1. Estimate the local MAP value for the site of interest. If the site is ungauged a spatial interpolation procedure can be used (see e.g., Fig. 2);

2. Estimate the index storm with duration $d, m_{d}$. If the site is ungauged a spatial interpolation procedure can be used (see e.g., Figs. 7-9; Sect. 5.2);

3. Compute the L-Cs $s_{R}$ as a function of the local MAP value, with model (6) and parameters $a, b$ and $c$ reported in Table 2;

4. Compute the L-Cv $v_{R}$ as a function of the local MAP value, with model (6) and parameters $a, b$ and $c$ reported in Table 2; 

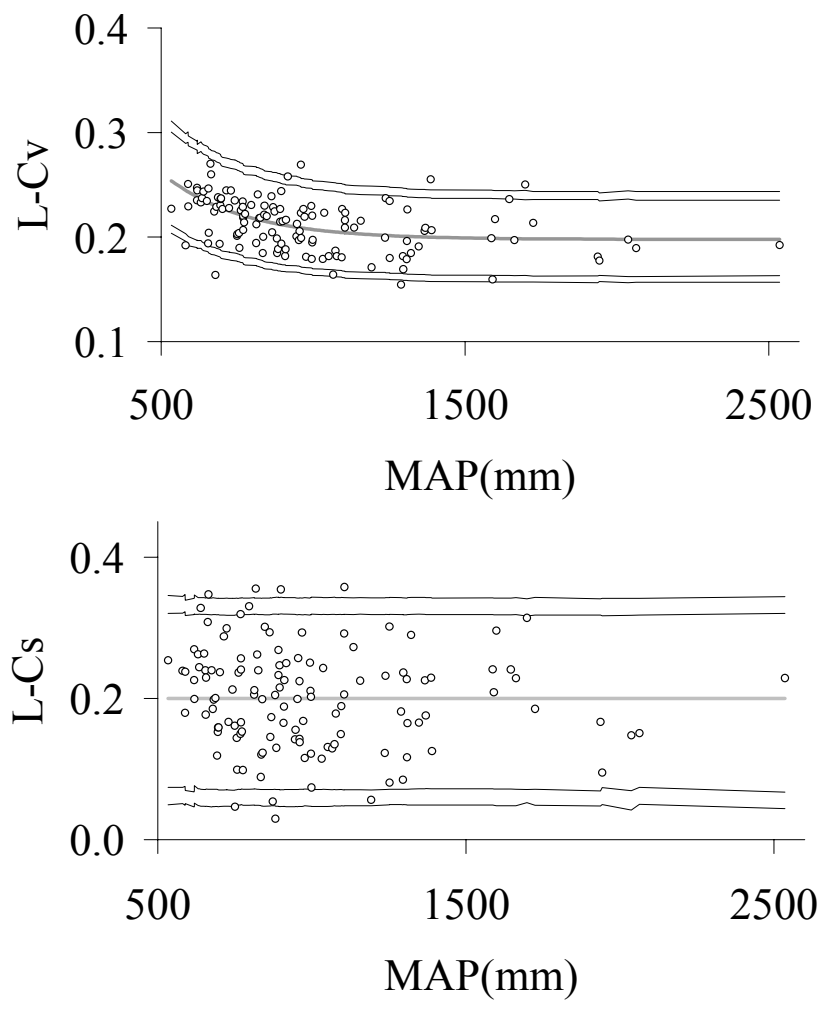

Fig. 6. Empirical models of $\mathrm{L}-\mathrm{Cs}_{R}$ and $\mathrm{L}-\mathrm{Cv}_{R}$ for $\mathrm{d}=1 \mathrm{~h}$ (grey lines); sample L-Cs and L- $C v$ (circles); 90 and $95 \%$ confidence intervals obtained trough Monte Carlo experiments (black lines).

5. Estimate the GEV parameters $\alpha^{\prime}, \xi^{\prime}$ and $k^{\prime}$, through Eqs. (5a) and (5b) using the $\mathrm{L}-C s_{R}$ and $\mathrm{L}-C v_{R}$ values estimated at steps $\mathrm{c}$. and d.;

6. Compute $h^{\prime}(d, T)$ using the parameters $\alpha^{\prime}, \xi^{\prime}$ and $k^{\prime}$ and the probability $\mathrm{F}$ equal to $1-1 / T$, where $T$ is the return period in years;

7. Compute an estimate of the design storm $h(d, T)$ as the product $h^{\prime}(d, T) m_{d}$.

\subsection{Index storm and MAP at ungauged sites}

The estimation of the design storm, for duration $d$, at ungauged sites requires an estimate of the index storm, $m_{d}$. This is a crucial step. The direct estimation of $m_{d}$ for an ungauged site is clearly impossible. Isoline maps of $m_{d}$ obtained with an adequate spatial interpolator, can be used instead (see e.g., Brath et al., 2003). Some isoline maps are reported in Figs. 7, 8 and 9. The indirect estimation of MAP is also essential for applying the proposed regional model to ungauged sites, as it clearly appears from Eq. (6). Also the local MAP value can be retrieved at ungauged sites from isoline maps. Therefore, indications on the uncertainty associated with standard spatial interpolation procedures are

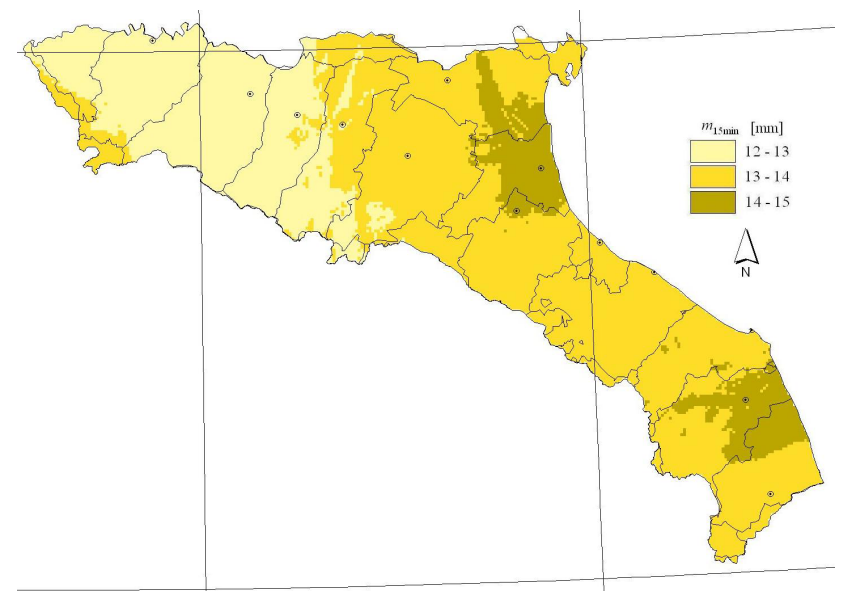

Fig. 7. Mean annual rainfall maxima for duration $d=15 \mathrm{~min}$.

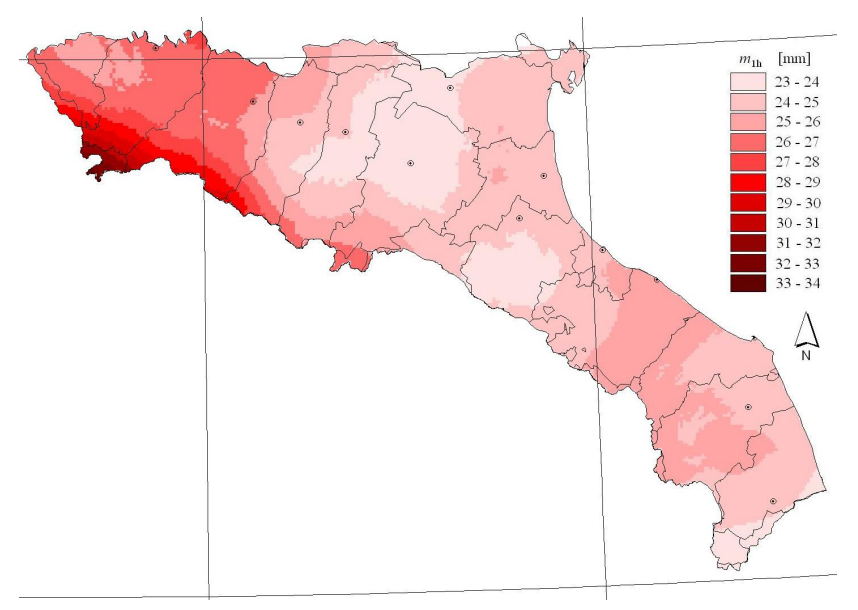

Fig. 8. Mean annual rainfall maxima for duration $d=1 \mathrm{~h}$.

very important. We report here the results of a series of resampling experiments aiming at assessing the reliability of $m_{d}$ and MAP estimates based upon isoline maps. The resampling experiments (jack-knife procedure, see Brath et al., 2003) are structured as follows:

1. we considered the duration $d$ and the number $N S$ of available raingauges where it was possible to calculate $m_{d}$ from the series of annual maximum depth with duration $d$ (sample mean of annual rainfall maxima);

2. one of these raingauges, say station i, and its corresponding $m_{d}$ value were removed from the set;

3. we generate a isopluvial map of $m_{d}$, interpolating the data of the remaining NS-1 raingauges sites;

4. a jack-knife estimate of $m_{d}$ for site $i$ was then retrieved from the map identified at step 3 ;

5. steps $2-4$ were repeated $N S-1$ times, considering in turn one of the remaining raingauges. 


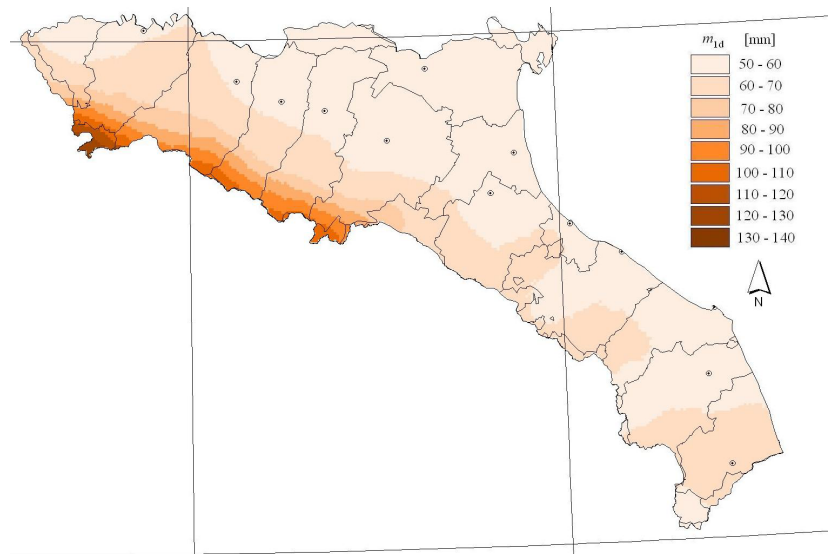

Fig. 9. Mean annual rainfall maxima for duration $d=1$ day.

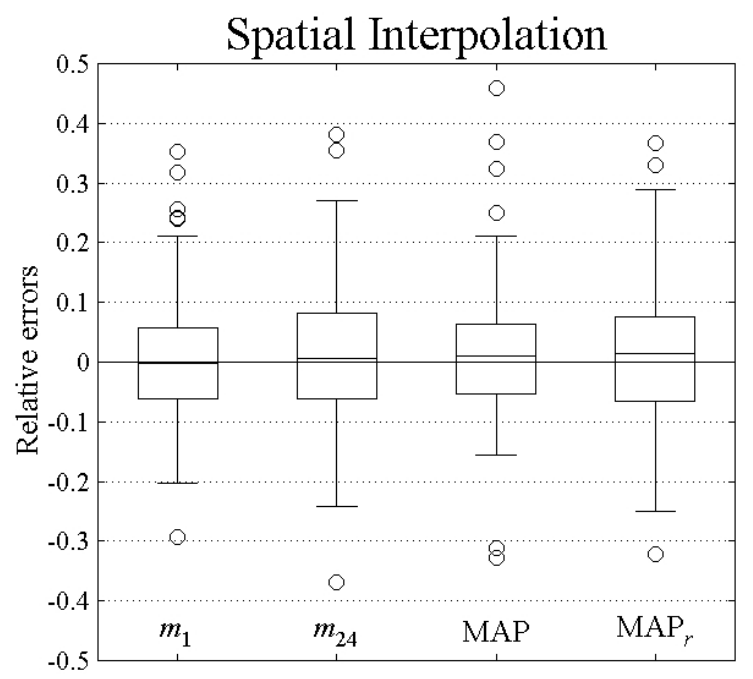

Fig. 10. Results of the cross-validation in terms of relative errors: box-plots summarize the relative errors distributions (values) in terms of 25th, 50th and 75th quantiles, maximum and minimum values and outliers (circles).

The NS empirical value of $m_{d}$ (i.e., sample means) were then compared with the corresponding values resulting from the jack-knife procedure (i.e. jack-knife estimates). The comparison allowed us to draw some considerations on the robustness and reliability of the spatial interpolation, through the following indexes of performance:

$$
\begin{aligned}
& \mathrm{BIAS}=1 / N S \sum_{i=1}^{N S}\left[\left(\hat{m}_{d, j k, i} \hat{m}_{d, i}\right) / \hat{m}_{d, i}\right] ; \\
& \mathrm{RMSE}=\sqrt{1 / N S \sum_{i=1}^{N S}\left[\left(\hat{m}_{d, j k, i} \hat{m}_{d, i}\right) / \hat{m}_{d, i}\right]^{2}}
\end{aligned}
$$

where the subscript $d, i$ indicates the sample estimate of $m_{d}$ for station $i$, which we assume to be exact, while the subscript $d, j k, i$ indicates the corresponding jack-knife estimate. This procedure can be applied several times, considering dif-
Table 4. RMSE and BIAS obtained for the estimation of rainfall indexes for 125 stations with at least 30 years of observation.

\begin{tabular}{llll}
\hline & KLV application & RMSE & BIAS \\
\hline$m_{1}$ & Empirical values & $11.2 \%$ & $1.0 \%$ \\
$m_{24}$ & Empirical values & $11.9 \%$ & $2.9 \%$ \\
MAP & Empirical values & $9.9 \%$ & $1.2 \%$ \\
MAP $_{r}$ & Relative Residuals of & $11.8 \%$ & $1.3 \%$ \\
& Regressive Model & & \\
\hline
\end{tabular}

ferent spatial interpolators, to identify the spatial interpolation method that has the minimum uncertainty.

The best performances, for estimating $m_{1}$ and $m_{24}$ ( $m_{d}$ for duration equal to $1 \mathrm{~h}$ and $24 \mathrm{~h}$ ), were obtained using ordinary kriging with linear variogram (KLV, Kitanidis, 1993), considering no more than 8 stations located at no more than 40 $\mathrm{km}$ from the site of interest. Table 4 shows the RMSE and BIAS values that were obtained estimating for 125 stations with at least 30 years of observation. The box-plot diagram of Fig. 10 shows the error distributions of relative errors of estimators $m_{1}$ and $m_{24}$. The reliability of local MAP values estimated from isoline maps of the study area was studied in an analogous way. We obtained the best results by the ordinary kriging with linear variogram.

Given that altitude is the factor that most affects MAP (see Fig. 2), we also considered, as a procedure for estimating MAP at ungauged sites, the ordinary kriging of relative residuals obtained from a suitable linear regression model between elevation and MAP. We tested several possible options as for regression models and variograms, and we obtained the best performance indexes after cross-validation for this particular case (i.e., ordinary kriging of relative residuals) by combining a linear regressive model for representing the relationship elevation-MAP, with a KLV spatial interpolation of relative residuals.

Table 4 shows the values of RMSE and BIAS obtained with a direct geographic interpolation of MAP (MAP) or an interpolation of the relative residuals of the regression model $\left(\mathrm{MAP}_{r}\right)$. The box-plots of Fig. 10 show the distributions of the relative errors for the two spatial interpolators.

The cross validation (i.e., resampling) procedure resulted in similar statistical indexes of performance for $m_{1}$ and $m_{24}$. In detail, the box-plots (Fig. 10) show rather high values of the maximum relative error (close to $40 \%$ ) and indicate relative errors bigger than $7-8 \%$ for about $50 \%$ of the estimates.

It is interesting to observe (Table 4 and Fig. 10) that the uncertainty of the ordinary kriging of relative residuals $\left(\mathrm{MAP}_{r}\right)$ is approximately the same, or inferior, than the uncertainty of ordinary kriging of the empirical MAP values (MAP). This indicates that taking explicitly into account the orographic effect on MAP values does not improve the estimation performances. 


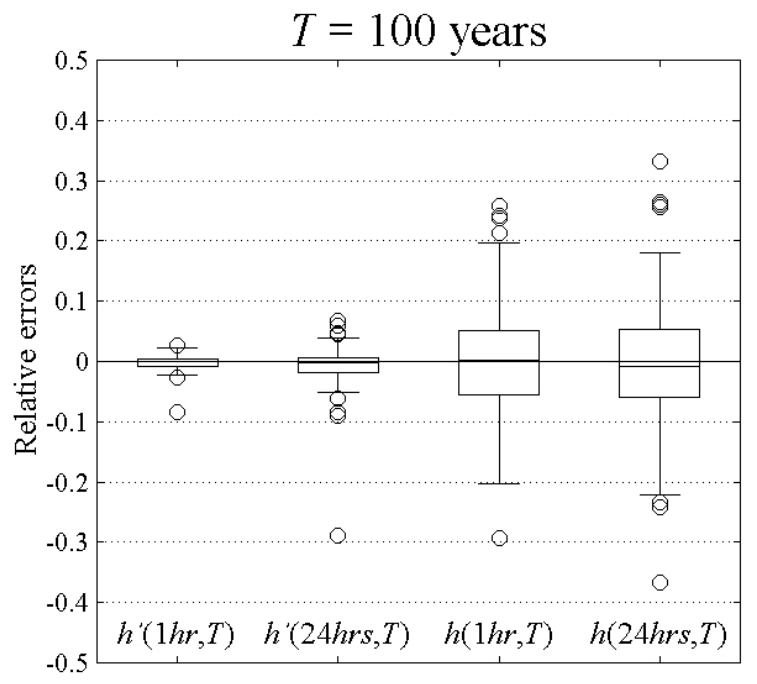

Fig. 11. Results of the cross-validation in terms of relative errors: box-plots summarize the relative errors distributions (values) in terms of 25th, 50th and 75th quantiles, maximum and minimum values and outliers (circles).

The results are obviously connected with the considered study area and raingauge network. Nevertheless, the analysis quantifies effectively (see Table 4 and Fig. 10) the uncertainty of $m_{d}$ and MAP from isoline maps, showing that the reliability level is analogous for all considered rainfall indexes (i.e., $m_{1}, m_{24}$ and MAP) and depends on the geostatistical spatial interpolator.

\subsection{Uncertainty of the regional estimates}

We evaluated the performance of the regional model through a comprehensive jack-knife cross-validation (see e.g., Brath et al., 2001). The cross-validation procedure enabled us to compare the regional and resampled estimates of the design storm at all considered raingauges for two arbitrarily selected duration: 1 and $24 \mathrm{~h}$ and two reference recurrence intervals: 100 and $200 \mathrm{yrs}$, which are normally adopted in Italy for designing flood risk mitigation measures. Through this comparison we quantified the uncertainty of the design storm estimates that can be computed by applying the proposed regional model to any ungauged site within the study area. In particular, we compared the regional estimate of the dimensionless growth factor, $h^{\prime}(d, T)$, and design storm, $h(d, T)$, with their resampled counterparts, $h_{j k}^{\prime}(d, T)$ and $\mathrm{h}_{j k}(d, T)$, respectively. We computed $h_{j k}^{\prime}(d, T)$ and $h_{j k}(d, T)$ as follows:

1. one of the NS raingauges, say station $i$, and its corresponding data were removed from the set;

2. parameters $a, b$ and $c$ of model (6) were estimated on the basis of the pluviometric information collected at the remaining NS-1 raingauges;

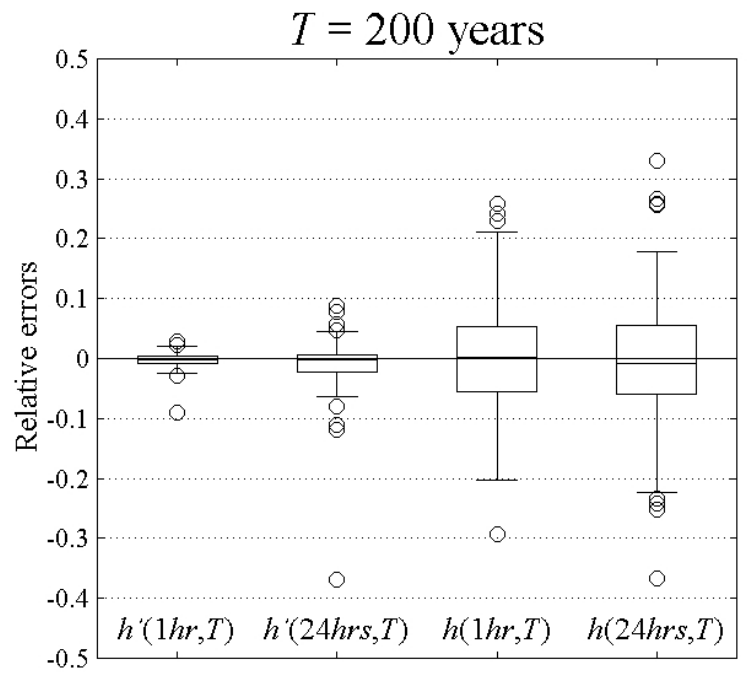

Fig. 12. Results of the cross-validation in terms of relative errors: box-plots summarize the relative errors distributions (values) in terms of 25 th, 50th and 75 th quantiles, maximum and minimum values and outliers (circles).

3. jack-knifed regional L-moments, $\mathrm{L}-C v_{R j k}$ and $\mathrm{L}-C s_{R j k}$, were calculated for site $i$ by using the recalibrated model (7) identified at step 2) and the jack-knifed MAP value $\left(\mathrm{MAP}_{j k}\right)$ retrieved for site $i$ from isoline MAP generated through ordinary kriging as described in Sect. 5.2;

4. the jack-knifed parameters of the regional GEV distribution were estimated for site $i$ through the method of $\mathrm{L}-$ moments on the basis of the $\mathrm{L}-C v_{\mathrm{Rjk}}$ and $\mathrm{L}-C s_{\mathrm{Rjk}}$ values estimated at step 3);

5. $h_{j k}^{\prime}(d, T)$ was computed for site i as the $T$-year quantile from the GEV distribution estimated at step 4);

6. $h_{j k}(d, T)$ was then computed as the product of $h_{j k}^{\prime}(\mathrm{d}, \mathrm{T})$ and the jack-knife estimate of $m_{d}$, which was calculated as described in Sect. 5.2;

7. steps $1-6$ were repeated NS- 1 times, considering in turn one of the remaining raingauges.

The box-plot diagram of Fig. 11 shows the distributions of relative errors for the estimation of the growth factor $h^{\prime}(1 \mathrm{~h}, 100 \mathrm{yrs})$ and $h^{\prime}(24 \mathrm{~h}, 100 \mathrm{yrs})$ and the design storm $h(1 \mathrm{~h}, 100 \mathrm{yrs})$ and $h(24 \mathrm{~h}, 100 \mathrm{yrs})$. Figure 12 shows the distributions of relative errors for the estimation of the growth factor $h^{\prime}(1 \mathrm{~h}, 200 \mathrm{yrs})$ and $h^{\prime}(24 \mathrm{~h}, 200 \mathrm{yrs})$ and the design storm $h(1 \mathrm{~h}, 200 \mathrm{yrs})$ and $h(24 \mathrm{~h}, 200 \mathrm{yrs})$. The figures show that the application of the proposed regional model to ungauged sites provides unbiased estimates of $h^{\prime}(d, T)$, for $d=1$, $24 \mathrm{~h}$ and T=100, $200 \mathrm{yrs}$. Also, Figs. 11 and 12 illustrate that 
the absolute value of relative errors of the dimensionless regional growth factors is generally small and always lower than $10 \%$.

It is important to notice that if the local value of MAP is known (e.g., from observed daily rainfall data), then the relative errors of $h^{\prime}(d, T)$ estimates, resulting from the uncertainties in the parameters of model (6), become practically negligible $(<1 \%)$.

Concerning the estimation of $h(d, T)$ the performance of the model is definitely lower than $30 \%$. A comparison between the relative errors of Figs. 11 and 12 points out rather clearly that the largest uncertainty in the application of the regional model to an ungauged site is associated with the index-storm estimates (see Sect. 5.2).

The presence of cross correlation between stations does not introduce a bias effect, but could increase the uncertainty of the estimates (see Hosking and Wallis, 1988). These effects are not quantified at this stage, but we are planning to investigate this matter in the future.

\section{Conclusions}

The paper presents a regional frequency analysis of annual maximum rainfall depths for storm duration ranging from $15 \mathrm{~min}$ to 1 day, observed for a dense network of raingauges placed in northern central Italy.

The study investigates the statistical properties of rainfall extremes using an approach based on L-moments, and detects important relations between these statistics and mean annual precipitation (MAP). Previous studies (Schaefer, 1990; Alila, 1999) showed that the statistics of rainfall extremes vary systematically with location and these studies also identified statistically significant relationships between these statistics and MAP, which was used as a surrogate of geographical location. For instance, Schaefer (1990) showed that the coefficients of variation and skewness of rainfall extremes tend to decrease as the local value of MAP increases. Our study confirmed in part these findings on a different geographic area.

We developed an empirical regional model that enables one to estimate the design storm in any location of the study area. We assessed the model applicability through Monte Carlo experiments and quantified the uncertainty of the estimates for ungauged sites using an extensive jack-knife resampling procedure.

The jack-knife procedure pointed out that the estimates of the design storm are basically unbiased and that the applicability of the regional model to ungauged sites is rather reliable. We obtained for the study area resampled estimates of the design storm characterised by relative errors generally lower that $10 \%$ and never higher than $40 \%$. Also, the resampling procedure highlighted that the highest uncertainty is associated with the estimation of the index storm (i.e., mean annual maximum rainfall depth for a given duration).
It is important to underline that the proposed regional model was developed through statistical optimisation. Therefore, that the model itself can be applied to storm duration from $15 \mathrm{~min}$ to 1 day and sites located within the study area. A careful application of the regional model should also consider that the model itself was developed for raingauges located below $1500 \mathrm{~m}$ a.s.l., while the altitude in the study area can locally exceed $2000 \mathrm{~m}$ a.s.l. Finally, the spatial interpolation of rainfall extremes or MAP adopted in our study is unable to reproduce micro-climatic effects such as rain shadow effects, and can only provide an overly simplified representation of differences existing between leeward and windward sides of the same mountain depending of the particular spatial interpolator adopted in the study.

\section{Appendix A}

\section{Homogeneity test}

The Hosking and Wallis test assesses the homogeneity of a group of basins at three different levels by focusing on three measures of dispersion for different orders of the sample Lmoment ratios (see Hosking (1990) for an explanation of Lmoments):

A measure of dispersion for the $\mathrm{L}-C v$ :

$V_{1}=\sum_{i=1}^{R} n_{i}\left(t_{2(i)}-\bar{t}_{2}\right)^{2} / \sum_{i=1}^{R} n_{i}$

A measure of dispersion for both the L-Cv and the L-Cs coefficients in the $\mathrm{L}-\mathrm{C} v$-L-Cs space:

$V_{2}=\sum_{i=1}^{R} n_{i}\left[\left(t_{2(i)}-\bar{t}_{2}\right)^{2}+\left(t_{3(i)}-\bar{t}_{3}\right)^{2}\right]^{1 / 2} / \sum_{i=1}^{R} n_{i}$

A measure of dispersion for both the L-Cs and the L-Ck coefficients in the L-Cs-L-Ck space:

$V_{3}=\sum_{i=1}^{R} n_{i}\left[\left(t_{3(i)}-\bar{t}_{3}\right)^{2}+\left(t_{4(i)}-\bar{t}_{4}\right)^{2}\right]^{1 / 2} / \sum_{i=1}^{R} n_{i}$

where $\bar{t}_{2}, \bar{t}_{3}$, and $\bar{t}_{4}$ are the group mean of $\mathrm{L}-C v, \mathrm{~L}-C s$, and L- $C k$ respectively; $t_{2(i)}, t_{3(i)}, t_{4(i)}$, and $n_{i}$ are the values of $\mathrm{L}-C v, \mathrm{~L}-C s, \mathrm{~L}-C k$ and the sample size for site $i$; and $R$ is the number of sites in the pooling group.

The underlying concept of the test is to measure the sample variability of the L-moment ratios and compare it to the variation that would be expected in a homogeneous group. The expected mean value and standard deviation of these dispersion measures for a homogeneous group, $\mu_{V_{k}}$ and $\sigma_{V_{k}}$ respectively, are assessed through repeated simulations, by generating homogeneous groups of basins having the same record lengths as those of the observed data following the methodology proposed by Hosking and Wallis (1993). 
The heterogeneity measures are then evaluated using the following expression:

$\mathrm{H}(k)=\frac{V_{k}-\mu_{V_{k}}}{\sigma_{V_{k}}} ;$ for $\quad k=1,2,3$

Hosking and Wallis (1993) suggested that the region or group of sites should be considered as acceptably homogeneous if $\mathrm{H}<1$; possibly heterogeneous if $1 \leq \mathrm{H}<2$, and definitely heterogeneous if $\mathrm{H} \geq 2$.

Acknowledgements. The study presented here has been carried out in the framework of the activity of the Working Group on the Characterisation of Ungauged Basins by Integrated uSe of hydrological Techniques (CUBIST). The work has been partially supported by the Italian Ministry of University and Research in Science and Technology (MURST). We would like to thank the Associate Editor, P. Molnar, and the Reviewers, P. Bernardara and Y. Alila, for providing very useful comments, which helped us in improving the presentation of our work.

Edited by: P. Molnar

\section{References}

Alila, Y.: A hierarchical approach for the regionalization of precipitation annual maxima in Canada, J. Geophys. Res., 104, 31 645$31655,1999$.

Brath, A. and Franchini, M.: La valutazione delle Pioggie intense su base regionale (in Italian), in: L'ingegneria naturalistica nella sistemazione dei corsi d'acqua, edited by: Maione, U. and Brath, A., BIOS (Cosenza), 65-91, 1999.

Brath, A. and Franchini, M.: la valutazione delle pioggie intense su base regionale (in Italian), Proceedings of the scientific seminar L'ingegneria naturalistica nella sistemazione dei corsi d'acqua, edited by: Maione, U. and Brath, A., BIOS (Cosenza), 65-91, 1999.

Brath, A. and Castellarin, A.: Tecniche di affinamento delle previsioni regionali del rischio pluviometrico (in Italian), Proceedings of the scientific seminar La progettazione della difesa idraulica Interventi di laminazione controllata delle piene fluviali, edited by: Maione, U., Brath, A., and Mignosa, P., BIOS (Cosenza), 93-121, 2001.

Brath, A., Castellarin, A., and Montanari, A.: Assessing the reliability of regional depth-duration-frequency equations for gaged and ungaged sites, Water Resour. Res., 39(12), 1367-1379, 2003.

Burlando, P. and Rosso, R.: Scaling and multiscaling DepthDuration-Frequency curves of storm precipitation, J. Hydrol., 187, 45-64, 1996.

Burn, D. H.: Evaluation of regional flood frequency analysis with a region of influence approach, Water Resour. Res., 26(10), 22572265,1990 .
Castellarin, A., Burn, D. H., and Brath, A.: Assessing the effectiveness of hydrological similarity measures for flood frequency analysis, J. Hydrol., 241(3-4), 270-285, 2001.

Castellarin, A. and Brath, A.: Tecniche di perfezionamento delle stime regionali del rischio pluviometrico (in Italian), Proceedings of the XXVIII Italian Conference on Hydraulics and Hydraulic Works, Potenza 16-19 settembre 2002, 1, 225-236, Ed. BIOS, Cosenza, 2002.

Castellarin, A. and Brath, A.: Descriptive capability of seasonality indicators for regional frequency analyses of flood and rainfall, Proceedings of the International Conference on Flood Estimation, CHR Rep. II-17, 429-440, Lelystad, Netherlands, 2002.

Dalrymple, T.: Flood frequency analysis, U.S. Geol. Surv. Water Supply Pap., 1543-A, 11-51, 1960.

Faulkner, D.: Rainfall frequency estimation, Flood estimation Handbook, 2, Wallingford, 1999.

Franchini, M. and Galeati, G.: La regionalizzazione delle pioggie intense mediante il modello TCEV. Una applicazione alla regione Romagna-Marche (in Italian), Idrotecnica, 5, 1994.

Gabriele, S. and Arnell, N.: A hierarchical approach to regional flood frequency analysis, Water Resour. Res., 27(6), 1281-1289, 1991.

Horton, R. E.: Analysis of runoff-plat experiments with varying infiltration capacity. Transactions, Am. Geophys. Union, 20, 693711, 1939.

Hosking, J. R. M.: L-moments: analysis and estimation of distributions using linear combination of order statistics, J. Royal Statistical Soc., Series B., 52(1), 105-124, 1990.

Hosking, J. R. M. and Wallis, J. R.: The Effect of Inter-Site Dependence on Regional Flood Frequency Analysis, Water Resour Res., 24, 588-600, 1988.

Hosking, J. R. M. and Wallis, J. R.: Some statistics useful in regional frequency analysis, Water Resour. Res., 29(2), 271-281, 1993.

Hosking, J. R. M. and Wallis, J. R.: Regional frequency Analysis, Cambridge University Press, New York, 1997.

Jenkinson, A. F.: The frequency distribution of the annual maximum (or minimum) of meteorological elements, Q. J. Royal Meteorol. Soc., 81, 1955.

Kitanidis, P. K.: Geostatistics, Handbook of Hydrology, edited by: Maidment, D. R., McGraw-Hill, New York, 20.1-20.39, 1993.

Klemes V.: Tall tales about tails of hydrological distributions: I and II, J. Hydrol. Eng., American Society of Civil Engineers 5(3), 227-239, 2000.

Marani, M.: On the correlation structure of continuous and discrete point rainfall, Water Resour. Res., 39(5), 1128, doi:10.1029/2002WR001456, 2003.

Olsson, J. and Burlando, P.: Reproduction of temporal scaling by a rectangular pulses rainfall model, Hydrol. Processes, 16, 611630, 2002.

Schaefer, M. G.: Regional analyses of precipitation annual maxima in Washington State, Water Resour. Res., 26(1), 119-131, 1990.

Schaefer, M. G.: Regional analyses of precipitation annual maxima in Washington State, Water Resour. Res., 26(1), 119-131, 1990. 